FedUni ResearchOnline https://researchonline.federation.edu.au

This is the peer-reviewed version of the following article:

Batool, M., Islam, S., Shahnia, F. (2017) Power transaction management amoungst coupled microgrids in remote areas. 7th IEEE Innovative Smart Grid Technologies - Asia, ISGT-Asia 2017;Auckland, New Zealand; 4th-7th December 2017 p. 1-6.

Which has been published in final form at:

https://doi.org/10.1109/ISGT-Asia.2017.8378449

Copyright @ 2017 IEEE. Personal use of this material is permitted. Permission from IEEE must be obtained for all other uses, in any current or future media, including reprinting/republishing this material for advertising or promotional purposes, creating new collective works, for resale or redistribution to servers or lists, or reuse of any copyrighted component of this work in other works. 


\section{Power Transaction Management amongst Coupled Microgrids in Remote Areas}

\author{
Munira Batool, Syed M. Islam \\ School of Electrical Engineering \& Computing \\ Curtin University \\ Perth, Australia \\ munirabatool@postgrad.curtin.edu.au; s.islam@curtin.edu.au
}

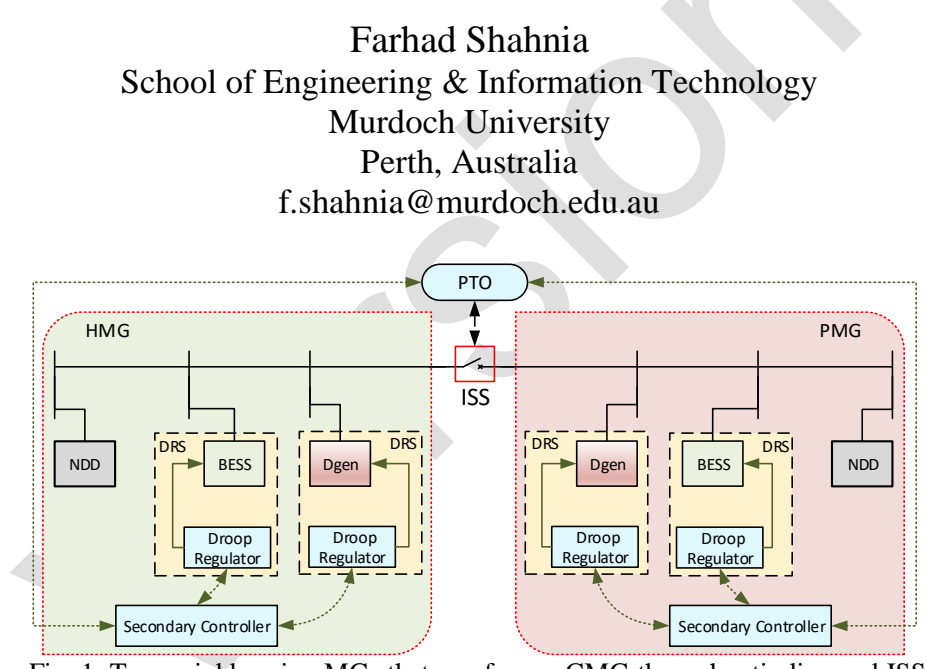

Fig. 1. Two neighbouring MGs that can form a CMG through a tie-line and ISS and with the help of the developed PTO.

other to increase their overall reliability, resilience and economics [3-4].

Recently, the concept of coupled MGs (CMGs) has been proposed in which two or more neighboring MGs of a remote area can interconnect provisionally to support each other during emergencies; e.g., when a fault occurs in a section of an MG, leading to the outage of one or some of its DERs, or when an MG is overloaded, or when an MG experiences excessive generation from its renewable energy-based non-dispatchable DERs (NDDs), or during normal conditions to minimize the levelized cost of electricity [5-7]. Let us consider the network of Fig. 1 which shows two neighboring MGs, connected through a tie-line and an interconnecting static switch (ISS). Under the concept of coupling the MGs, an MG observing the emergency condition, referred to as the problem MG (PMG) can be supported by an available and healthy MG (HMG) provisionally.

A transformative architecture is proposed for coupling the nearby MGs in [8] to improve the system resiliency during faults. A decision-making-based approach is proposed in [9] to determine the most suitable HMG(s) to be coupled with an overloaded PMG, which considers different criteria such as available surplus power, electricity cost, reliability and the distance of the neighboring MGs as well as the voltage/frequency deviation in the CMG. Ref. [7] presents the conditions based on which the overloading of a PMG and the availability of excess power in the neighboring HMG can be detected. Ref. [10] presents an interac- and grid-connected mode (when a utility feeder is available) [12]. A cluster of remote area MGs are assumed to have physical connections amongst themselves that they can use to support each
Remote area microgrids (MGs) are considered as selfcontrolled electrical system with penetration of distributed energy resources (DERs) for better reliability and reduced costs. They can operate in stand-alone (also referred to as off-grid or isolated)

\begin{abstract}
Large remote areas normally have isolated and self-suf-
ficient electricity supply systems, often referred to as microgrids.
These systems also rely on a mix of dispatchable and non-dispatchable distributed energy resources to reduce the overall cost of electricity production. Emergencies such as shortfalls, overloading, and microgrids. This paper presents a power transaction management scheme amongst a few such microgrids when they are coupled provisionally during emergencies. By definition, power transaction is an
instance of buying and selling of electricity amongst problem and healthy microgrids. The developed technique aims to define the suitpower transaction amongst the coupled microgrids. To this end, an optimization problem is formulated that aims to define the above painteger linear programming technique is used to solve the formulated problem. The performance of the proposed management strat-
\end{abstract}

BESS

DER

MG

PMG

Problem microgrid

\section{INTRODUCTION}

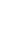
Index Terms-Droop regulated system, Coupled microgrids, OptimiSOC 
tive control of CMGs to guarantee adequate load sharing and system-wide stability. The dynamic operation of DERs within CMGs is investigated in [11] whereas [12] examines the dynamic security of the CMGs. The interaction among the DERs of the MGs in a CMG is investigated in [13]. Ref. [14] analyses the reliability aspects of a CMG while their voltage and current controllability, as well as small signal stability, are analyzed in [15-17]. References [18-19] present a technique to coordinate the operation of BESS in MGs along with their provisional coupling. Coupling of MGs can be realized by back-to-back converters [20] or by ISSes [7] between the adjacent MGs. The ultimate vision is that an MG can be interconnected to any MG (and not necessarily an adjacent MG) if a general link is available to act as a power exchange highway. References [21-22] presents an optimization-based technique to coordinate the CMGs while in [23-25], the calculation of least operation cost solution, using different optimization techniques, is discussed in details for CMGs. In [26-27], it is shown that CMGs can work in cooperative mode in case of high penetration of NDDs in the network while they provide robust distributed control.

This paper proposes the power transaction management scheme among MGs of a CMG following any unexpected emergency condition. The NDDs and loads are assumed to be uncontrolled while the droop regulated system (DRS) such as the diesel gas or diesel-based generator (Degn) and the battery energy storage systems (BESSs) inside each MG, which adopt voltage and frequency droop control, serve as the control variables. Frequency adjustment is not needed if the MG is operating in grid-connected mode, but assuming a remote area without a utility feeder, the dispatchable DERs are assumed responsible for the frequency regulation. The NDDs operate under a constant PQ control mode. Moreover, a low bandwidth communication is assumed available to control the power flow in an MG and its synchronization with neighboring $\mathrm{MG}(\mathrm{s})$.

\section{THE CONCEPT}

Consider Fig. 1 illustrating two neighboring MGs with physical links among each other which can facilitate their temporary interconnection during emergency conditions. A power transaction operator (PTO) is considered with the following responsibilities:

- receiving information from secondary controllers of each MG,

- identifying a PMG,

- solving an optimization problem to select a suitable neighboring HMG to exchange power with and define the level of power transaction (import/export), and

- transmitting the decision variables to the secondary controllers of each MG of a CMG.

In such a concept, the operation of the DRSs includes the following steps:

- transmitting the data to PTO through MG secondary controller,

- determining the operational set-points for DRSs based on the received information from $\mathrm{PTO}$, via the MG secondary controller.

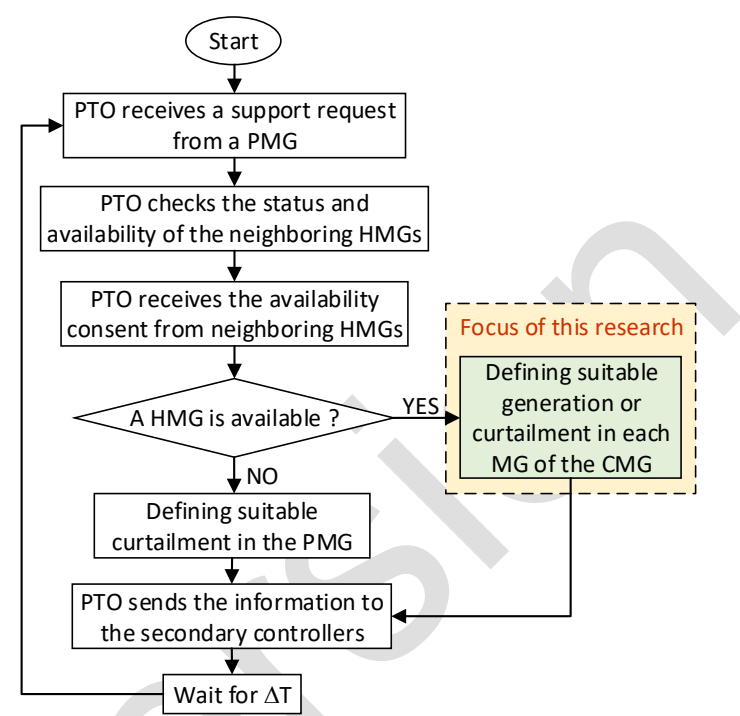

Fig. 2. Flow chart of the power transaction strategy in which the focus of this research is highlighted.

The formulation of the problem to decide the selection of HMG(s) along with the desired constraints is as described below: After receiving the information of emergencies from a PMG, the PTO check the availability of a neighboring HMG. If a HMG is found available to support the PMG, the PTO will solve an optimization problem to determine the suitable power transaction in the tie-lines within the desired CMG. If a HMG is not available or no feasible solution is found through solving the optimization problem, the PTO will send a command to the PMG to proceed with curtailing either of the consumption of its non-essential loads or the generation output of its NDDs. The PTO is assumed active all the time and responds instantaneously to any changes in the MGs. It also reevaluates the system conditions in $\Delta T$ intervals. The operational principle of the PTO is illustrated schematically in the flowchart of Fig. 2.

In the CMG network of Fig. 1, one MG is defined as the power exporting one while the other is assumed as the power importing one in an emergency condition. It is also assumed that the exchanged power (denoted by $P_{\text {trans }}$ ) is positive if a power flow is observed from the HMG to the PMG and negative if vice versa. Now, the deviation between the planned transacted power (i.e., the optimized PTO output) and the actual value (before the optimization) can be expressed as

$\Delta P^{\text {trans }}=P_{\text {new }}^{\text {trans }}-P_{\text {old }}^{\text {trans }}$

where $\Delta P^{\text {trans }}$ represents the required power transacted between neighboring MGs within the CMG while subscripts new and old respectively denote the power flow in the tie-line after and before the optimization. To adjust the power transaction by each MG, the equal generation costs incremental principle, described in [28], is considered. Now, suppose following a sudden demand increase in an MG, it becomes the PMG. Thus, the PTO aims at increasing the power flow from the HMG to the PMG to accommodate the 
extra demand. The power transaction should not rise above a certain limit, so according to the equal incremental cost principle, the transacted cost incremental rate will rise up to $\lambda_{i x}$ for the corresponding output power of DRS be $P_{\text {exp-ix }}^{\mathrm{HMG}}$. Thereby, the sum of changes of the output power of the DRS will be equal to the total power exported or imported from/to the HMG, i.e.,

$\sum \Delta P_{k}^{\text {Dgen }}+\sum \Delta P_{k}^{\text {BESS }}=\Delta P_{\exp }$

This can be formulated with an objective function (OF) such as

$$
\left.\begin{array}{rl}
O F=\sum_{M G=1}^{N}\left(\sum_{k}(\right. & \left(C^{\text {fuel }}+C^{\text {cfp }} \partial_{k}\right)\left|\Delta P_{k}^{\text {Dgen }}\right| \\
& +C_{\text {lifeloss }}^{\text {BESS }}\left|\Delta P_{k}^{\text {BESS }}\right|+C_{\text {curt }}^{\text {NDDs }}\left|\Delta P^{\text {NDDs }}\right| \\
& \left.+C_{\text {curt }}^{\text {load }}\left|\Delta P^{\text {load }}\right|+C^{\text {trans }}\left|\Delta P^{\text {trans }}\right|\right) \text { Time }
\end{array}\right)
$$

which consists of four part: The first part aims to minimize the cost (denoted by $C$ ) of a change in power of DRSs. For diesel generators (denoted by ${ }^{\text {Dgen}}$ ), it includes the cost for fuel and carbon footprints (denoted by ${ }^{\text {cfp }}$ ) where $\partial$ is the emission factor. As the BESS does not observe any fuel costs, only the cost of its life loss change is considered. The third and fourth parts correspond to the required curtailment in the consumption of non-essential loads and the generation output of NDDs. The last part refers to the change in power transaction cost amongst MGs of the CMG. The Time factor is included in (3) to estimate the total time required for power transaction to overcome emergency condition in PMGs (which is equal to $\Delta T$ of Fig. 2). Also, the index $k$ illustrates the number of Dgens and BESSs in each MG. The considered constraints, when solving (3), can be expressed by

$$
\begin{aligned}
& P_{\text {DER }}^{k}=P_{\text {load }}^{k}+V^{i} \sum_{j=1}^{N} V^{j}\left(G^{i j} \cos \left(\theta^{i j}\right)+B^{i j} \sin \left(\theta^{i j}\right)\right) \\
& \left(P_{\text {Dgen }}^{k}\right)^{\text {min }} \leq P_{\text {Dgen }}^{k} \leq\left(P_{\text {Dgen }}^{k}\right)^{\max } \\
& \left(P_{\mathrm{BESS}}^{k}\right)^{\min } \leq P_{\mathrm{BESS}}^{k} \leq\left(P_{\mathrm{BESS}}^{k}\right)^{\max } \\
& V^{\min } \leq V^{k} \leq V^{\max } \\
& f^{\min } \leq f \leq f^{\max } \\
& I_{l} \leq I_{l}^{\max } \\
& S o C^{\min } \leq S o C^{k} \leq S o C^{\max }
\end{aligned}
$$

Eq. (4) is the power balance equation in which $i$ and $j$ are the bus index for an MG, while $V$ and $\theta$ are respectively the voltage amplitude and phase at each bus, whereas $G$ and $B$ are respectively the real and imaginary parts of the row $i$ and column $j$ of the admittance matrix. Constraints (5)-(6) are the expected output power limit for the Dgens and BESSs while constraints (7)-(8) are the voltage and frequency deviation limits. Constraint (9) is the thermal current limit of each line while constraint (10) is the SOC limit of the BESSs.

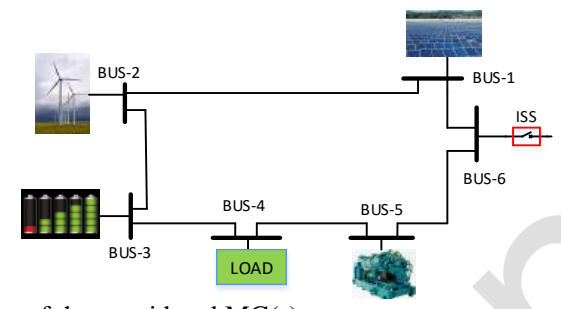

Fig. 3. Topology of the considered MG(s)

Table 1. Considered input data for the numerical analyses.

\begin{tabular}{c|c|cc|cccc}
\hline NDDs & Load & \multicolumn{2}{|c|}{ Dgen } & \multicolumn{4}{c}{ BESS } \\
\hline $\begin{array}{c}\boldsymbol{P}_{\text {NDDs }}^{\text {cap }} \\
(\mathrm{kW})\end{array}$ & $\begin{array}{c}P_{\text {load }}^{\text {cap }} \\
(\mathrm{kW})\end{array}$ & $\begin{array}{c}\mathrm{P}_{\text {Dgen }}^{\min } \\
(\mathrm{kW})\end{array}$ & $\begin{array}{c}\mathrm{P}_{\text {Dgen }}^{\max } \\
(\mathrm{kW})\end{array}$ & $\begin{array}{c}\text { Capacity } \\
(\mathrm{kWh})\end{array}$ & $\begin{array}{c}\mathrm{SOC}_{\text {LIMIT }} \\
(\%)\end{array}$ & $\begin{array}{c}\mathrm{P}_{\text {BESS }}^{\text {CH,MAX }} \\
(\mathrm{kW})\end{array}$ & $\begin{array}{c}\mathrm{P}_{\text {BESS }}^{\text {DCH,MIN }} \\
(\mathrm{kW})\end{array}$ \\
\hline 95 & 100 & 3.0 & 110 & 50 & $30-90$ & 48 & 5 \\
\hline
\end{tabular}

Table 2. Considered costs data for the numerical analyses.

\begin{tabular}{lc|ll}
\hline $\boldsymbol{C}^{\text {fuel }}$ & $0.31 \$ / \mathrm{kWh}$ & $\boldsymbol{C}_{\text {curt }}^{\text {load }}$ & $0.15 \$ / \mathrm{kWh}$ \\
\hline $\boldsymbol{C}^{\text {cfp }}$ & $0.02 \$ / \mathrm{kg}$ & $C_{\text {lifeloss }}^{\text {BESS }}$ & $0.98 \$ / \mathrm{kWh}$ \\
\hline $\boldsymbol{C}_{\text {curt }}^{\mathrm{NDDs}}$ & $0.3 \$ / \mathrm{kWh}$ & $C^{\text {trans }}$ & $0.4 \$ / \mathrm{kWh}$ \\
\hline$\partial$ & $0.003 \mathrm{~kg} / \mathrm{kWh}$ & & \\
\hline
\end{tabular}

Table 3: Distance of each MG from central common point

\begin{tabular}{ccccccc}
\hline & MG-1 & MG-2 & MG-3 & MG-4 & MG-5 & MG-6 \\
\hline Distance $(\mathrm{km})$ & 4 & 6 & 2 & 7 & 5 & 5 \\
\hline
\end{tabular}

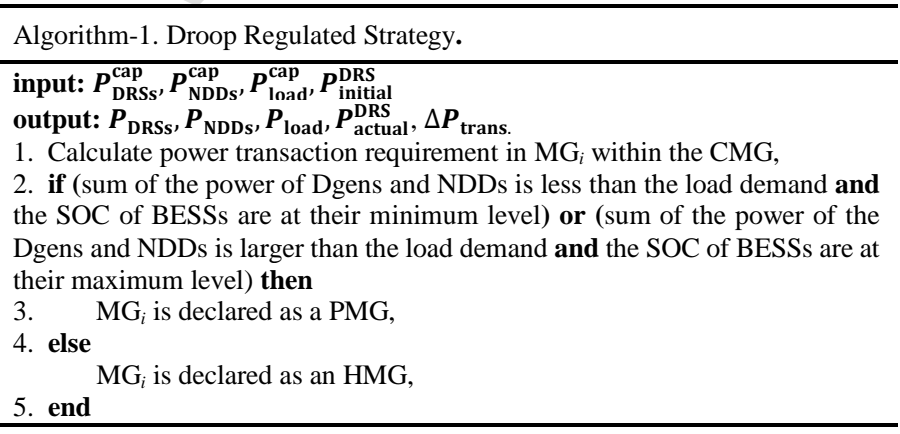

\section{PERFORMANCE EVALUATION}

The formulated optimization problem is assessed using the mixed-integer linear programming technique. Exhaustive simulations are carried out in MATLAB to evaluate the developed power transaction strategy. A system of $6 \mathrm{MGs}$ are considered, as shown in Fig. 3. As the internal structure of the MGs is not important in the developed strategy, for simplicity, all MGs are assumed to have the same topology in the numerical analyses of this paper. The impedance data for all of MGs buses is taken from [9]. The pre-defined input data assumed for all participating MGs and the cost data are listed in Table 1 and 2 while Table 3 shows the distance of each considered MG from a central node at which they are coupled. First, the HMG(s) and PMG(s) are identified in each study using Algorithm-1. Once the status of participating MGs are known, the optimization technique will determine the output power of the DRSs, the power curtailment of non-essential loads or generation output of NDDs in each MG of the CMG, as well as the change in power transaction. Based on these calculations and 
the data provided in Table 2, the $O F$ of (3) is calculated. Based on the minimum $O F$, the $\mathrm{CMG}$ with the optimal operating conditions is defined.

The developed technique is evaluated on the considered system with thousands of repetitions in which the powers of DRS, NDDs and loads are created randomly in a stochastic environment. The acceptable limits of voltage and frequency in this study are $1 \pm 0.075 \mathrm{pu}$ and $50 \pm 0.5 \mathrm{~Hz}$ respectively. The developed power transaction strategy is validated through several study cases, a few of which discussed below.

\section{A) Study Case-1:}

Consider an event in which one of the MGs (i.e., MG-5) with a total demand of $65 \mathrm{~kW}$ is overloaded (see Fig. 4). Only $13.5 \mathrm{~kW}$ is supplied by the NDDs. The system has a maximum voltage magnitude of $0.994 \mathrm{pu}$ (which is below the nominal voltage) and a frequency of $49.29 \mathrm{~Hz}$ (which is below the minimum desired frequency of $49.5 \mathrm{~Hz}$ ). The SoC of the BESS is also assumed to be $38 \%$ and thus, its discharging can cause the violation of SOC limits. A total power shortfall of $9.8 \mathrm{~kW}$ is observed in this MG. Using algorithm-1, the PTO declares MG-5 as a PMG and then uses the mixed-integer linear programming technique to solve the $O F$ of (3) for the system under this condition. The optimization defines that the best alternative (i.e., the most economical solution) is to import power from two HMGs of MG-2 and MG-4 simultaneously. The value of $O F$ for the system is calculated as 9.003\$ with MG-2 and MG-4 exporting respectively 4.9 and 5.5 $\mathrm{kW}$ of power (out of which $0.2 \mathrm{~kW}$ is wasted in the form of power line losses). As a result, the minimum and maximum voltage magnitude and the frequency observed in the CMG system composed of these three MGs are respectively $0.989 \mathrm{pu}, 1.034 \mathrm{pu}$ and 50.21 $\mathrm{Hz}$. So in this way the emergency situation (i.e., the overloading of MG-5) an be mitigated by only importing power from other neighboring MGs while no load curtailment is observed for the overloaded MG.

\section{B) Study case-2:}

Consider another event in which MG-3 observes an excessive generation from its NDDs (see Fig. 5). The demand of the MG is $12 \mathrm{~kW}$ while the generation from NDDs and Dgen is $14.3 \mathrm{~kW}$. The system observes a maximum voltage of $1.076 \mathrm{pu}$ and a frequency of $49.55 \mathrm{~Hz}$ (both above the acceptable limits). Moreover, the SoC of the BESS is already reached the maximum level and cannot absorb the extra available excess power from the NDDs. In this situation, the PTO declares MG-3 as a PMG using algorithm-1 and proceeds to apply the optimization technique to find the best solution. As a result, it determines that MG-1 (which has with maximum voltage of $1.035 \mathrm{pu}$ and frequency as $49.72 \mathrm{~Hz}$, a demand of $35.2 \mathrm{~kW}$ out of which $9.3 \mathrm{~kW}$ is supplied by the NDDs) as a suitable HMG with an $O F$ value of $7.4328 \$$. Thus, it is suggested to couple MG-1 with MG-3 and form a CMG in which MG-3 exports $1.5 \mathrm{~kW}$ power, out of which $1.4 \mathrm{~kW}$ is injected to MG-1 (due to power line losses). In addition, MG-3 curtails its
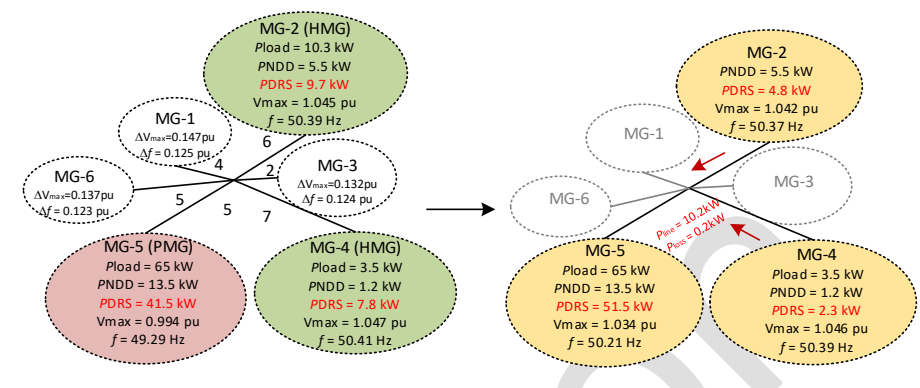

Fig. 4. Considered study case-1.

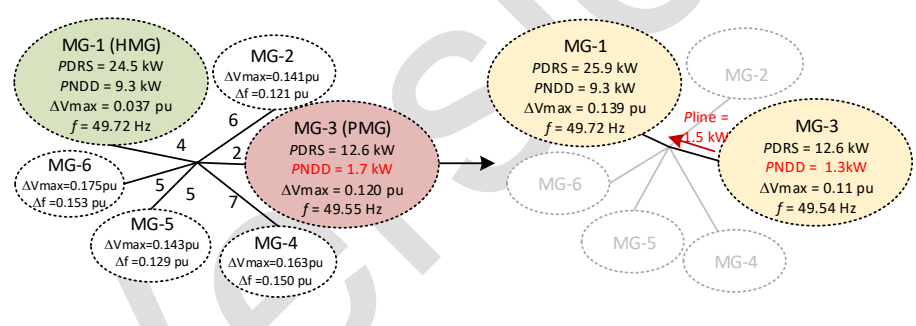

Fig. 5. Considered study case-2.

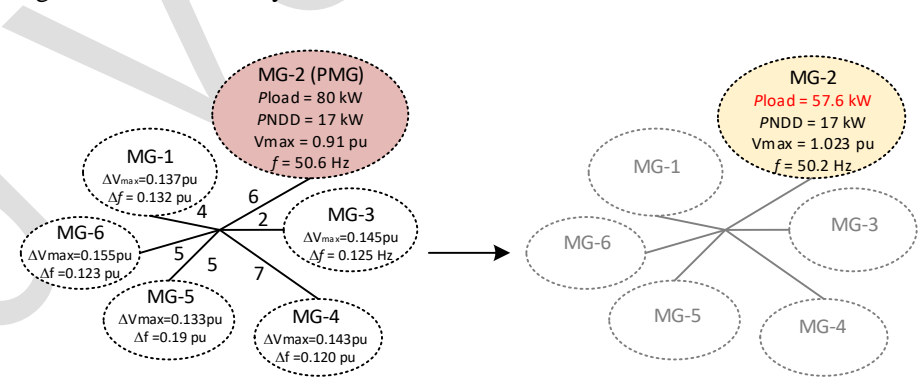

Fig. 6. Considered study case-3.

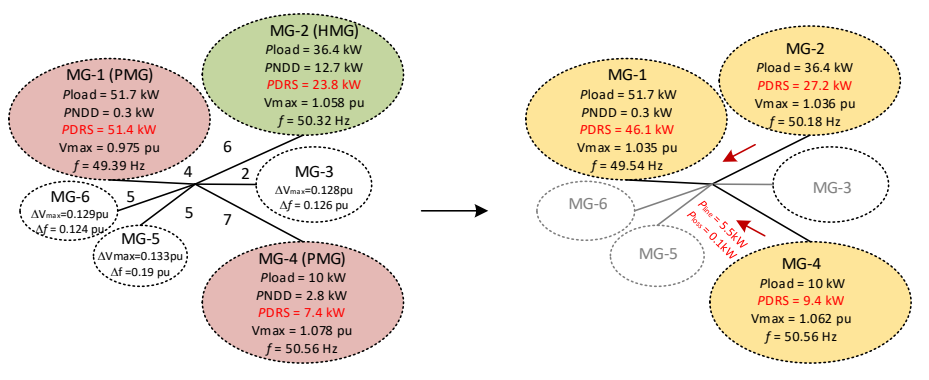

Fig. 7. Considered study case-4.

NDDs by $0.4 \mathrm{~kW}$.

C) Study Case-3:

Consider another event in which MG-2 is overloaded and its maximum voltage magnitude is $0.91 \mathrm{pu}$, which is not within the acceptable limit. The demand of this MG is as-sumed to be $80 \mathrm{~kW}$, while the sum of power supplied by Dgens and NDDs are 65.5 $\mathrm{kW}$ out of which $17 \mathrm{~kW}$ is supplied by the NDDs. The SoC of the BESS is assumed to be at the lowest level, thus making it unable 
to supply the power shortfall. Thereby, using algorithm-1, the PTO defines the most optimal solution is to proceed with a loadshedding of $19 \mathrm{~kW}$ and without any power transaction with other neighboring MGs due to technical constraints. The value of $O F$ will be $4.2678 \$$. As a result, MG-2 will reduce its load by $19 \mathrm{~kW}$ and the thus, the Dgen will supply only $39.2 \mathrm{~kW}$ while the rest comes from the NDDs. Also, the maximum voltage gets improved to $1.023 \mathrm{pu}$.

D) Study case-4: Consider another event in which more than one MG is declared as PMG (see Fig. 7). In this example, MG-1 is overloaded with a load demand of $51.7 \mathrm{~kW}$ while the NDDs contribution is only $0.3 \mathrm{~kW}$. Thereby, the Dgen generates 51.4 $\mathrm{kW}$ and the BESS has reached to its maximum discharge level. As a result, the frequency drops down to $49.39 \mathrm{~Hz}$ (i.e., below the minimum acceptable range of frequency). On the other hand, MG-4 experiences excessive generation. Its load demand is only $10 \mathrm{~kW}$ out of which $2.8 \mathrm{~kW}$ is supplied by NDDs while the Dgen's contribution is $7.4 \mathrm{~kW}$. The BESS is not active. Thus, the frequency is $50.56 \mathrm{~Hz}$ while maximum voltage is $1.078 \mathrm{pu}$ (both above the maximum acceptable range). In this situation, PTO declares both MG-1 and MG-4 as PMG using algorithm-1. It then uses the optimization technique to look for the available options in order to choose the most economical power transaction strategy. MG-2 is a HMG with $36.4 \mathrm{~kW}$ load and nominal frequency and voltage of $50.32 \mathrm{~Hz}$ and 1.058 pu respectively. As a result MG-1, MG-2 and MG-4 are coupled together while MG-2 and MG-4 export a total power of $5.5 \mathrm{~kW}$ to MG-1 out of which 5.4 $\mathrm{kW}$ is injected in MG-1 (due to line loss of $0.1 \mathrm{~kW}$ ). The $O F$ value is calculated as 7.3462\$. Due to this action, the emergency condition of overloading and excessive generation is resolved in both PMGs and they retain their frequency and voltage values and settle down as normal operating MGs in the considered remote area.

\section{CONCLUSION}

The main aim of the developed power transaction strategy is to enable the optimal power flow amongst provisionally coupled neighboring MGs within a remote area, in which no utility feeder is available. The considered emergency situations include overloading or excessive generation from renewable-based NDDs. The considered PTO is active all the time and runs in specific intervals to guarantee the CMG's optimal operation at lowest costs and minimal technical impacts. The developed algorithm defines the optimal output power of DRSs in all MGs within the CMG, the curtailment of non-essential loads and power generation of renewable-based NDDs and sends the outputs to the secondary controllers of each provisionally connected MG to apply them in their system. The performance of the developed technique and its efficiency in defining the optimal operation points has been validated by a stochastic analysis in MATLAB.

\section{REFERENCES}

[1] L. Che, and M. Shahidehpour, "DC microgrids: Economic operation and enhancement of resilience by hierarchical control," IEEE Trans. SmartGrid, vol.5, no.5, pp.2517-2526, 2014.
[2] L. Che, M. Khodayar, and M. Shahidehpour, "Only connect: Microgrids for distribution system restoration," IEEE Power Energy Magazine, vol.12, no.1, pp.70-81, 2014

[3] P.C. Loh, L. Ding, Y. Chai, and F. Blaabjerg, "Autonomous operation of hybrid microgrid with ac and dc subgrids," IEEE Trans. Power Electronics, vol.28, no.5, pp.2214-2223, 2013.

[4] L. Che, M. Shahidehpour, A. Alabdulwahab, and Y. Al-Turki, "Hierarchical coordination of a community microgrid with ac and dc microgrids," IEEE Trans. Smart Grid, vol.6, no.6, pp. 3042-3051, 2015.

[5] R.H. Lasseter, "Smart distribution: coupled microgrids," Proceedings of the IEEE, vol.99, no.6, pp.1074-1082, 2011.

[6] Z. Wang, B. Chen, J. Wang, and C. Chen, "Networked microgrids for selfhealing power systems," IEEE Trans. Smart Grid, vol.7, no.1, pp.310-319, 2016.

[7] E. Pashajavid, F. Shahnia, and A. Ghosh, "Development of a self-healing strategy to enhance the overloading resilience of islanded microgrids," IEEE Trans. Smart Grid, vol.8, no.2, pp.868-880, 2017.

[8] Z. Wang, and J. Wang, "Self-healing resilient distribution systems based on sectionalization into microgrids," IEEE Trans. Power Systems, vol.30, no.6, pp.3139-3149, 2015.

[9] F. Shahnia, S. Bourbour, and A. Ghosh, "Coupling neighboring microgrids for overload management based on dynamic multicriteria decision-making," IEEE Trans. Smart Grid, vol.8, no.2, pp.969-983, 2017.

[10] Y. Zhang, L. Xie, and Q. Ding, "Interactive control of coupled microgrids for guaranteed system-wide small signal stability," IEEE Trans. Smart Grid, vol.7, no.2, pp.1088-1096, 2016.

[11] F. Shahnia, R.P.S. Chandrasena, S. Rajakaruna, and A. Ghosh, "Primary control level of parallel distributed energy resources converters in system of multiple interconnected autonomous microgrids within self-healing networks,” IET Gen. Tran. \& Dist., vol.8, pp.203-222, 2014.

[12] Y. Zhang, and L. Xie, "Online dynamic security assessment of microgrid interconnections in smart distribution systems," IEEE Trans. Power Systems, vol.30, no.6, pp.3246-3254, 2015.

[13] I.P. Nikolakakos, H.H. Zeineldin, M.S. El-Moursi, and N.D. Hatziargyriou, "Stability evaluation of interconnected multi-inverter microgrids through critical clusters," IEEE Trans. Power Systems, vol.31, no.4, pp.3060-3072, 2016.

[14] N. Nikmehr, S.N. Ravadanegh, "Reliability evaluation of multi-microgrids considering optimal operation of small scale energy zones under loadgeneration uncertainties," International Journal of Electrical Power \& Energy Systems, vol.78, pp.80-87, 2016.

[15] S.A. Arefifar, M. Ordonez, and Y. Mohamed, "Voltage and current controllability in multi-microgrid smart distribution systems," IEEE Trans. Smart Grid, doi: 10.1109/TSG.2016.2568999.

[16] F. Shahnia, and A. Arefi, "Eigenanalysis-based small signal stability of the system of coupled sustainable microgrids," International Journal of Electrical Power \& Energy Systems, vol.91, pp.42-60, 2017.

[17] F. Shahnia, "Stability and eigenanalysis of a sustainable remote area microgrid with a transforming structure," Sustainable Energy, Grids \& Networks, vol.8, pp.37-50, 2016.

[18] F. Shahnia, "Semi-decentralized charging and discharging control of floating batteries in microgrids," $2^{\text {nd }}$ IEEE Annual Southern Power Electronics Conference (SPEC), pp.1-6, Auckland, 2016.

[19] T.H. Mehr, A Ghosh, and F. Shahnia, "Cooperative control of battery energy storage systems in microgrids," International Journal of Electrical Power \& Energy Systems, vol.87, pp.109-120, 2017.

[20] R. Majumder, and G. Bag, "Parallel operation of converter interfaced multiple microgrids," International Journal of Electrical Power \& Energy Systems, vol.55, pp.486-496, 2014.

[21] A. Arefi, and F. Shahnia, "Tertiary controller-based optimal voltage and frequency management technique for multi-microgrid systems of large remote towns," IEEE Trans. Smart Grid, doi:10.1109/TSG.2017.2700054.

[22] F. Shahnia, and A. Arefi, "Defining the suitable adjacent microgrids to form a temporary system of coupled microgrids," IEEE Region 10 Conference (Tencon), pp.1-4, Singapore, 2016.

[23] J. Wang, M. Shahidehpour, and Z. Li, "Strategic generation capacity expansion planning with incomplete information," IEEE Trans. Power Syst., 
vol.24, no.2, pp.1002-1010, 2009

[24] Y. Tohidi, F. Aminifar, and M. Fotuhi-Firuzabad, "Generation expansion and retirement planning based on the stochastic programming," Elect. Power Syst. Res., vol.104, pp.138-145, 2013.

[25] M. Fathi, and H. Bevrani, "Statistical cooperative power dispatching in interconnected microgrids," IEEE Trans. Sustainable Energy, vol.4, no.3, pp.586-593, 2013.

[26] H. Wang, and J. Huang, "Cooperative planning of renewable generations for interconnected microgrids", IEEE Trans. Smart Grid, vol.7, no.5, pp.24862496, 2016
[27] M.J. Hossain, M.A. Mahmud, F. Milano, et.al., "Design of robust distributed control for interconnected microgrids", IEEE Trans. Smart Grid, vol.7, no.6, pp.2724-2735, 2016.

[28] F. Chen, M. Chen, Q. Li, et.al., "Cost-based droop schemes for economic dispatch in islanded microgrids", IEEE Trans. Smart Grid, vol.8, no.1, pp.63-74, 2017. 